\title{
ARTICLE
}

\section{Evaluation of the neutron response of a criticality accident alarm system detector}

\author{
Norio Tsujimura ${ }^{*}$ and Tadayoshi Yoshida \\ Japan Atomic Energy Agency, 4-33 Muramatsu, Tokai-mura, Naka-gun, Ibaraki-ken, 319-1194, Japan
}

\begin{abstract}
A neutron irradiation experiment was performed to determine the neutron absorbed dose rate response of a criticality accident alarm system detector. For this purpose, the authors tailored a neutron field by the use of a ${ }^{252} \mathrm{Cf}$ neutron source and 50-mm-thick lead blocks, which removed unwanted gamma rays emitted from the source itself while allowing the neutrons to pass through. The evaluated neutron response of the detector was confirmed to be in reasonably good agreement with the prior computer-predicted response.
\end{abstract}

Keywords: criticality accident alarm system; response; neutron absorbed dose; ${ }^{252} \mathrm{Cf}$; lead filter

\section{Introduction}

A criticality accident alarm system (CAAS) is a purpose-built radiation monitor intended to limit the personnel dose received from a criticality accident by detection and immediate area evacuation. The Nuclear Fuel Cycle Engineering Laboratories (NCL) of the Japan Atomic Energy Agency (JAEA) has used such systems for over 40 years, and 30 systems are currently operational at the site. The latest model of the CAAS recently developed uses a plastic scintillator combined with a cadmium converter, and thus is sensitive both to gamma rays and neutrons [1]. Prior to the system installation in 2009, a series of detector testing exposed to gamma-ray sources were conducted. However, for neutron irradiation testing, the presence of unwanted gamma rays in neutron fields was a complication because the response of the CAAS due to the neutron component of a mixed neutron-gamma field is indistinguishable from that caused by the photon dose.

This study aims to determine a reasonably reliable neutron response of the CAAS detector by experiments and verify the computed response generated at the design stage of the detector. The neutron source required for this study would need to provide a neutron field with a sufficiently small gamma-to-neutron ratio and a neutron absorbed dose rate of the order of a few $\mathrm{mGy} / \mathrm{h}$ in the steady-state. To achieve this, a lead filtration technique proposed by Attix [2] was used here to filter out gamma rays from a ${ }^{252} \mathrm{Cf}$ neutron field while allowing the neutrons to pass. A description is given of a temporally constructed neutron field composed of a ${ }^{252} \mathrm{Cf}$ source and lead blocks. This paper also describes the result of the CAAS detector's neutron response obtained from the irradiation testing at this field.

*Corresponding author. Email: tsujimura.norio@jaea.go.jp

\section{Criticality Accident Alarm System}

The CAAS consisted of a group of three detector units, a logic unit composed of three two out of three voting units, an alarm unit that actuates an area evacuation warning throughout the facility, and power supplies and subsidiary modules [1]. The detector unit tested in this study consists of a specially designed radiation detector and related electronics circuitry and a power unit assembled in a steel box with height, width, and depth of 22,50 , and $24 \mathrm{~cm}$, respectively. The detector is a cylindrical plastic scintillator, $38 \mathrm{~mm}$ in diameter and $51 \mathrm{~mm}$ in length, coupled with a photomultiplier tube, all of which is placed into a 50-mm-thick polyethylene moderator. Dual layers of cadmium incorporated into the moderator generate gamma rays by ${ }^{113} \mathrm{Cd}(n, \gamma)$ reaction, which boosts the neutron sensitivity of the central plastic scintillator. The optimal configuration of the moderator and cadmium converters was accomplished by the use of a modern computer code for the detector to be capable of proportionally responding to total absorbed doses, regardless of whether it was exposed to gamma rays, neutrons, or a combination of both [1].

The anode current of the photomultiplier tube is converted to a voltage by an operational amplifier, and then the output voltage is supplied to a comparator, which provides an alarm trip signal to a two out of three voting unit when the output exceeds a preset value. The logic unit actuates alarm sounds and warning lights when at least two detector units out of the three provide alarm trip signals within a coincidence time of $500 \mathrm{~ms}$. The output of the operational amplifier is normally adjusted to $1 \mathrm{~V}$ as the constant live background, and any incremental output of $\sim 0.7 \mathrm{~V}$ corresponds to a tissue absorbed dose rate of $1 \mathrm{mGy} / \mathrm{h}$. After the completion of performance testing at a pulse radiation field, the CAAS 
was installed at the Tokai Reprocessing Plant of the NCL and has been in service since 2009.

\section{Method}

\subsection{Facility description}

Neutron irradiation testing was conducted at the irradiation room No. 4 of the Facility for Radiation Standards (FRS) of the Nuclear Science Research Institute, the JAEA. It is a large, concrete-enclosed cell measuring $12.5 \mathrm{~m} \times 12.5 \mathrm{~m} \times 11.7 \mathrm{~m}(\mathrm{H})$ with a steel grating floor at the mid-height of the room [3]. $\mathrm{A}{ }^{252} \mathrm{Cf}$ source with a nominal activity of $2 \mathrm{GBq}$ was used; the neutron emission rate was $1.77 \times 10^{8} \mathrm{n} / \mathrm{s}$ at the time of the experiment (Oct. 27, 2008). The neutron source was, when not in use, stored in a storage vault adjacent to the cell. When in use, it was carried by a remote trolley to the center of the ground floor and then delivered onto the tip of a source-holding rod attached to a lifter which is a guide rail affixed to an elevator tower that extends to the steel grating mid-floor. A winch mechanism moved the lifter to the mid-floor; in the fully-up position, the source anchored to the source-holding rod of the lifter passed through a $30-\mathrm{cm}$ square opening of the mid-floor and then reached the irradiation position $1 \mathrm{~m}$ above the floor. The total source transit time was $\sim 30 \mathrm{~min}$.

\subsection{Experimental set-up}

A ${ }^{252} \mathrm{Cf}$ source spontaneously emits unwanted gamma rays with an average energy of $\sim 0.8 \mathrm{MeV}$ and fission neutrons with an average energy of $2.1 \mathrm{MeV}$. Measurements were therefore made in the following two configurations: bare, uncollided ${ }^{252} \mathrm{Cf}$ and ${ }^{252} \mathrm{Cf}$ filtered by a 50 -mm-thick lead. The latter configuration was intended to achieve a pure neutron field with a reduced gamma-ray component. Six lead blocks, each measuring $50 \times 100 \times 200 \mathrm{~mm}^{3}$, were temporally erected to form a 50 -mm-thick wall with a $100 \times 100 \mathrm{~mm}^{2}$ vertical center channel, through which the ${ }^{252} \mathrm{Cf}$ source was introduced from below. Lead was selected as the attenuator material in the construction because it reduces the gamma-ray components without significantly modifying the neutron spectrum. Another reason for selecting the $50 \mathrm{~mm}$ thickness is that a $50-\mathrm{mm}$ block size was readily available and it is also greater than the one-tenth value thickness of $\sim 40 \mathrm{~mm}$ for $1 \mathrm{MeV}$ gamma rays.

Figure 1 shows the experimental set-up. The lead wall surrounding the ${ }^{252} \mathrm{Cf}$ source was built on an aluminum plate of $50 \times 60 \times 1 \mathrm{~cm}^{3}$ supported by a stand constructed of two-by-four lumber. The effective center of the detector unit, the center of the cylindrical plastic scintillator, was placed $35 \mathrm{~cm}$ from the uncollided ${ }^{252} \mathrm{Cf}$ source and $38 \mathrm{~cm}$ from the lead-filtered ${ }^{252} \mathrm{Cf}$ source, with the cylinder axis perpendicular to the source. These distances were chosen to achieve the reference neutron tissue absorbed dose rate of $1-2 \mathrm{mGy} / \mathrm{h}$ without causing any interference between the support tables. The output voltage of the operational amplifier of the detector unit was routed through a maze to a control room by twisted shielded pair cables and was repeatedly read 10 times by a digital multimeter with a 5-s time interval, and the net readings exceeding the background were averaged.

In addition, a reference photon dosemeter using a neutron-insensitive Geiger-Muller (GM) counter was also placed at the same distance but in a direction opposite to the detector unit, as shown in Figure 1. The GM counter was operated with a scaler timer, and all irradiations were performed with the anode perpendicular to the radiation field gradient. A detailed description of this counter is given in Section 3.3.
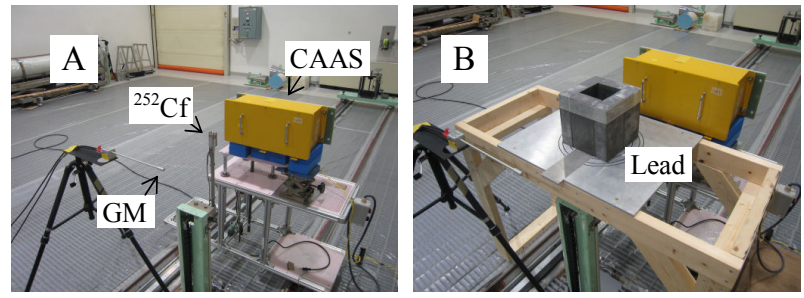

Figure 1. Experimental set-up: (A) uncollided ${ }^{252} \mathrm{Cf}$; (B) lead-filtered ${ }^{252} \mathrm{Cf}$. The CAAS detector (back) and the GM detector were placed equidistant from the source.

\subsection{Reference dosimetry}

The GM counter, the Model GM-1, was used to measure the gamma-ray component of the absorbed dose rate in the neutron fields and was manufactured by Far West Technology, Inc. (An identical instrument is discussed in a companion article in this issue [4].) The counter was equipped with a composite metal shield to flatten the gamma-energy dependence and a lithium compound shield to reduce the thermal neutron response; the former shield yielded a relatively flat air-kerma response over a wide energy range from 70 $\mathrm{keV}$ to a few $\mathrm{MeV}$. The neutron absorbed dose response of this counter was estimated to be less than $1 \%$ of the gamma response. The overall performance of this counter in a mixed neutron-gamma field was confirmed by Yoshida et al [4]. Prior to the test, a ${ }^{137} \mathrm{Cs}$ gamma-ray source was used to calibrate the counter.

The reference values of the neutron absorbed dose rate were derived from the neutron source intensity on the date of the measurement and the neutron energy spectra at each point of the test. The latter was calculated by the Monte Carlo code MCNP-4C2 [5] in conjunction with the JENDL-3.2 cross-section library [6]. The calculation model included a stainless steel source capsule, an outer stainless steel holder, a source-holding electromagnet, lead blocks and an aluminum plate. The construction details of the source holder were taken from the literature [7]; the electromagnet was approximated to be a steel cylinder with a diameter of $20 \mathrm{~mm}$ and length of $40 \mathrm{~mm}$ with the preservation of the actual mass of $60 \mathrm{~g}$. The concrete walls, the steel grating floor, air, etc. were not included in the model. The source neutron spectrum of ${ }^{252} \mathrm{Cf}$ was represented by a Maxwellian with a nuclear temperature of $1.42 \mathrm{MeV}$. Neutron spectral fluences at $35 \mathrm{~cm}$ from 
the bare ${ }^{252} \mathrm{Cf}$ source and at $38 \mathrm{~cm}$ from the lead-filtered ${ }^{252} \mathrm{Cf}$ source were both tallied by the point (F5) tally function in the MCNP, folded with the fluence-to-ambient absorbed dose $\left(D^{*}(10)\right)$ conversion coefficients [8], and finally multiplied by the source strength to yield the absorbed dose rate at each point of test. $D^{*}(10)$ was adopted here because it is for stationary monitors and by definition removes the quality factor accounting for a long-term stochastic risk.

\section{Results and discussion}

\subsection{Reference neutron and photon dose rate}

Figure 2 shows a comparison of the neutron spectra between the lead-filtered ${ }^{252} \mathrm{Cf}$ and the ideal scatter-free ${ }^{252} \mathrm{Cf}$. The quantity plotted is $E \phi(E)$, where $\phi(E)$ is the fluence/MeV at energy $E$, and both plots of the neutron spectra are normalized to the unit area to facilitate comparison. The spectrum of neutrons penetrating through the lead was slightly degraded by inelastic scattering; this resulted in a neutron spectrum with a lower mean energy of $1.6 \mathrm{MeV}$. No neutron loss by the absorption reactions within the lead blocks and surroundings was observed; therefore, a neutron dose intensity nearly equivalent to that with no blocks was expected to be delivered.

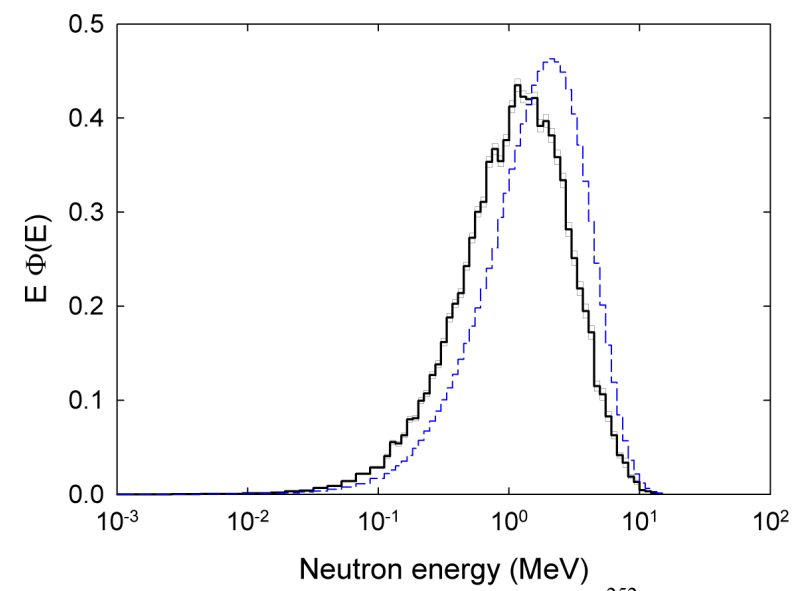

Figure 2. Calculated neutron spectrum of the ${ }^{252} \mathrm{Cf}$ filtered by a 50-mm-thick lead block. An ideal scatter-free ${ }^{252} \mathrm{Cf}$ spectrum is also shown in dashed line for comparison.

Table 1 presents the computed ambient absorbed dose rate of neutrons $\left(D_{\mathrm{n}}\right)$, and the gamma-ray air-kerma rate $\left(K_{\gamma}\right)$ and ambient absorbed dose rate $\left(D_{\gamma}\right)$ both measured by the GM counter. The latter two quantities include the secondary gamma rays generated in the lead itself. The conversion from air-kerma to the gamma-ray ambient absorbed dose, both in units of Gy, was taken as 1.2, based on the study of Yoshida and coworkers [4]. With the uncollided ${ }^{252} \mathrm{Cf}$ source, the dose rate values at $38 \mathrm{~cm}$, corrected by the inverse-square law, are also given in parentheses for comparison with the lead-filtered ${ }^{252} \mathrm{Cf}$. The comparison revealed that the 50-mm-thick lead shield reduced the gamma dose rate by a factor of $\sim 10$, while only cutting $5 \%$ of the neutron dose. Presented in the rightmost column is the absorbed dose ratio of $D_{\gamma} / D_{\mathrm{n}}$, varying from 0.06 to 0.4 . The neutron field with a smaller $D_{\gamma} / D_{\mathrm{n}}$ ratio, i.e., the lead-filtered ${ }^{252} \mathrm{Cf}$, was expected to provide a more suitable test condition.

Table 1. Summary of the reference neutron and gamma-ray dosimetry in the test neutron field.

\begin{tabular}{cccccc}
\hline Source & $\begin{array}{c}L \\
(\mathrm{~cm})\end{array}$ & $\begin{array}{c}D_{\mathrm{n}} \\
(\mathrm{mGy} / \mathrm{h})\end{array}$ & $\begin{array}{c}K_{\gamma} \\
(\mathrm{mGy} / \mathrm{h})\end{array}$ & $\begin{array}{c}D_{\gamma} \\
(\mathrm{mGy} / \mathrm{h})\end{array}$ & $D_{\gamma} / D_{\mathrm{n}}$ \\
\hline Uncollided & 35 & 1.5 & 0.54 & 0.65 & 0.4 \\
& $(38)$ & $(1.3)$ & $(0.46)$ & $(0.55)$ & \\
Lead, $50 \mathrm{~mm}$ & 38 & 1.2 & 0.05 & 0.06 & 0.06 \\
\hline
\end{tabular}

$L=$ Source-to-detector distance.

\subsection{Neutron response of the CAAS detector}

The output reading, $M$, of the CAAS detector to a mixed gamma + neutron field is $M=R_{\gamma} \times D_{\gamma}+R_{\mathrm{n}} \times D_{\mathrm{n}}$, where $D_{\gamma}$ and $D_{\mathrm{n}}$ represent the gamma and neutron dose rates in units of $\mathrm{Gy} / \mathrm{h}$, given in Table 1 , and $R_{\gamma}$ and $R_{\mathrm{n}}$ represent the detector responses per unit absorbed dose rate of gamma rays and neutrons, respectively. Thus, the neutron response is derived by $R_{\mathrm{n}}=\left(M-R_{\gamma} \times D_{\gamma}\right) / D_{\mathrm{n}}$.

Table 2 summarizes the test results. The $R_{\gamma} \times D_{\gamma}$ value, corresponding to the CAAS detector reading attributable to the accompanying gamma-ray components, was deduced because the CAAS detector had a gamma-response similar to that of the GM counter in a gamma-ray field with a predominance of several hundred $\mathrm{keV}$ gamma rays. The uncertainty of $R_{\gamma} \times D_{\gamma}$ was estimated to be $\pm 20 \%$, accounting for the calibration uncertainty of the GM counter and the systematic difference of the energy dependence of the two detectors. As a result of the neutron irradiation testing, the neutron absorbed dose rate responses were determined to be 0.29 $( \pm 23 \%)$ and $0.34( \pm 2 \%) \mathrm{V} /(\mathrm{mGy} / \mathrm{h})$ to the uncollided and the slightly degraded fission neutrons, respectively. The small ratio of $D_{\gamma} / D_{\mathrm{n}}=0.06$ observed in the latter neutron field contributed to the reduction of the propagation of uncertainties.

Table 2. Evaluated neutron absorbed dose rate response of the CAAS detector.

\begin{tabular}{ccccc}
\hline Source & $\begin{array}{c}M \\
(\mathrm{~V})\end{array}$ & $\begin{array}{c}R_{\gamma} \times D_{\gamma} \\
(\mathrm{V})\end{array}$ & $\begin{array}{c}M-R_{\gamma} \times D_{\gamma} \\
(\mathrm{V})\end{array}$ & $\begin{array}{c}R_{\mathrm{n}} \\
(\mathrm{V} /(\mathrm{mGy} / \mathrm{h}))\end{array}$ \\
\hline Uncollided & 0.87 & 0.44 & 0.43 & 0.29 \\
& \pm 0.01 & \pm 0.09 & \pm 0.10 & $\pm 23 \%$ \\
Lead, & 0.45 & 0.04 & 0.41 & 0.34 \\
$50 \mathrm{~mm}$ & \pm 0.01 & \pm 0.01 & \pm 0.01 & $\pm 2 \%$ \\
\hline
\end{tabular}

Figure 3 exhibits the energy dependence of the relative neutron absorbed dose rate response of the CAAS detector to the neutrons of normal incidence. Two experimental data were plotted at the neutron energy averaged over the spectrum, while the computer-calculated response [9] was for monoenergetic neutrons greater than $1 \mathrm{eV}$ and thermal neutrons with a Maxwellian velocity distribution at room temperature. 
The neutron response is normalized to the dose rate response of $0.67 \mathrm{~V} /(\mathrm{mGy} / \mathrm{h})$ to ${ }^{137} \mathrm{Cs}$ gamma rays; therefore, an ordinate of 0.1 means that the response to 1 $\mathrm{mGy} / \mathrm{h}$ of neutrons is equal to one-tenth of the response to $1 \mathrm{mGy} / \mathrm{h}$ of ${ }^{137} \mathrm{Cs}$ gamma-rays. A comparison showed that experimental results reasonably agreed with those obtained calculations, with the former plots systematically lying over the latter ones. This discrepancy is probably due to two reasons. First, the reference neutron dose rate did not include the roomand air-scattered neutron dose components, and second, the CAAS detector actually responded to such scattered low-energy neutrons with increased sensitivity. Both reasons led to the overestimation of the neutron response of the CAAS detector to fission neutrons in the experiments.

The computer-predicted response at the design stage was utilized to set the detection threshold of the dose rate forecast in a possible criticality accident at the NCL Tokai Reprocessing Plant. Therefore, the reasonably good agreement observed between the experimental and calculated responses to fission neutrons provided strong experimental support to the calculated response functions over the entire energy range from thermal to a few $\mathrm{MeV}$.

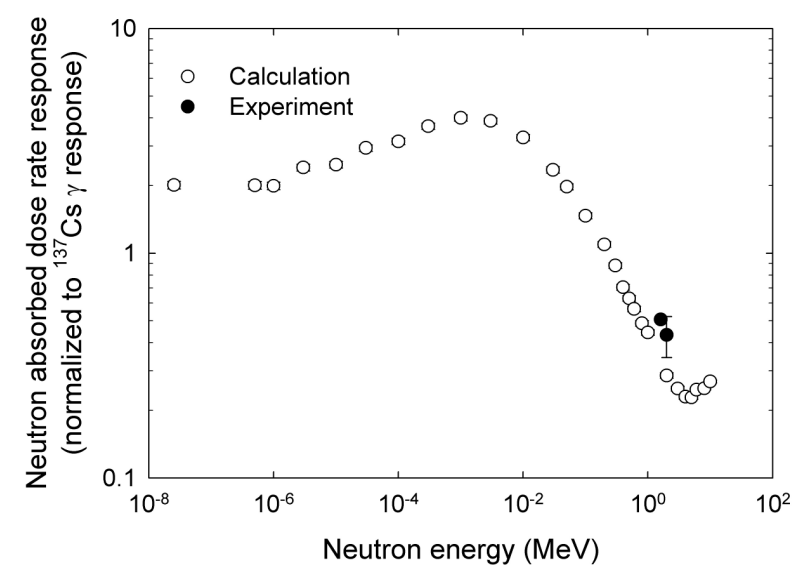

Figure 3. Neutron absorbed dose rate response of the CAAS detector as a function of the neutron energy. Closed circles, experiments; open circles, computed response [9].

\section{Conclusion}

A neutron irradiation experiment was performed to determine the neutron absorbed dose rate response of a criticality accident alarm system detector. For this purpose, the authors tailored a neutron field by the use of a ${ }^{252} \mathrm{Cf}$ neutron source and 50-mm-thick lead blocks, which removed unwanted gamma rays emitted from the source itself while allowing the neutrons to pass through. The neutron response experimentally obtained was confirmed to be in good agreement with the computer-predicted response generated at the design stage of the detector. The result has provided credibility to the basis used in setting the detection threshold of the dose rate forecast for a possible criticality accident at the NCL Tokai Reprocessing Plant.

\section{Acknowledgements}

The authors wish to thank Messrs K. Kawasaki and I. Onuma for their assistance in arranging the experiments and operating the neutron irradiation system at the FRS.

\section{References}

[1] N. Tsujimura and T. Yoshida, New criticality accident alarm system detectors at the JAEA Tokai Reprocessing Plant, Prog. Nucl. Sci. Technol. 1 (2011), pp. 202-205.

[2] R. H. Attix, R. B. Theus and C. C. Rogers, Measurement of dose components in an $n-\gamma$ field, Proc. 2nd Symposium on Neutron Dosimetry in Biology and Medicine, EUR-5273, (1975), pp. 329-336.

[3] M. Kowatari, K. Fujii, M. Takahashi, M. Yoshizawa, S. Simizu, K. Kawasaki and Y. Yamaguchi, Evaluation of the characteristics of the neutron reference field using $\mathrm{D}_{2} \mathrm{O}$-moderated ${ }^{252} \mathrm{Cf}$ source, Radiat. Prot. Dosim. 126 (2007), pp. 138-144.

[4] T. Yoshida, N. Tsujimura and H. Miyata, Measurement of photon dose rate in moderated neutron calibration fields, Prog. Nucl. Sci. Technol. (this issue).

[5] J. F. Briesmeister (Ed.), MCNP - A General Monte Carlo N-Particle Transport Code, LA-13709-M, Los Alamos National Laboratory, (2000).

[6] T. Nakagawa, K. Shibata, S. Chiba, T. Fukahori, Y. Nakajima, Y. Kikuchi, T. Kawano, Y. Kanda, T. Ohsawa, H. Matsunobu, M. Kawai, A. Zukeran, T. Watanabe, S. Igarashi, K. Kosako and T. Asami, Japanese Evaluated Nuclear Data Library Version 3 Revision 2, J. Nucl. Sci. Technol. 32 (1995), pp. 1259-1271.

[7] J. R. Dumais, M. Yoshizawa and Y. Yamaguchi, Evaluation of Scattered Neutrons in Fast Neutron Calibration Field using ${ }^{252} \mathrm{Cf}$ and ${ }^{241} \mathrm{Am}-\mathrm{Be}$ Neutron Source, JAERI-Tech 98-005, Japan Atomic Energy Research Institute, (1998). [in Japanese]

[8] B. R. L. Siebert and H. Schuhmacher, Calculated fluence-to-directional and personal dose equivalent conversion coefficients for neutrons, Radiat. Prot. Dosim. 54 (1994), pp. 231-238.

[9] N. Tsujimura, Determination of energy and angular dependencies of the neutron-gamma criticality accident detector, Annual Report on the Present State and Activities of the Radiation Protection Department, Nuclear Fuel Cycle Engineering Laboratories in Fiscal 2007, JAEA-Review 2008-071, Japan Atomic Energy Agency, (2009), pp. 75-78. [in Japanese] 